\title{
MICROENCAPSULAÇÃO DO EXTRATO DE JAMBO: PLANEJAMENTO EXPERIMENTAL E POTENCIAL DE APLICAÇÃO
}

\author{
A. L. M. L. da MATA ${ }^{1}$, J. L. MAIA ${ }^{1}$ e B. L. de A. $\operatorname{COSTA}^{1}$, C. G. de $\operatorname{MEDEIROS}^{1}$ B.P. da COSTA \\ $\mathrm{NETO}^{1}$ \\ ${ }^{1}$ Universidade Federal do Rio Grande do Norte, Departamento de Engenharia Química \\ E-mail para contato: juleaom@hotmail.com
}

\begin{abstract}
RESUMO - A microencapsulação tem sido usada na indústria de alimentos para fornecer materiais estáveis. As antocianinas possuem alto potencial antioxidante, entretanto são fotodegradáveis. Os desafios são direcionados à busca de técnicas que façam com que este potencial permaneça ativo e biodisponível. Este trabalho tem como objetivo propor a técnica de polimerização interfacial para encapsular as antocianinas presentes no extrato do jambo vermelho. Um planejamento experimental do tipo $2^{\mathrm{k}}$ fatorial foi realizado a fim de definir as melhores condições do processo. Foram definidas as condições ideais de temperatura e agitação do sistema. As micropartículas obtidas foram caracterizadas quanto ao tamanho, morfologia e carga superficial. Os resultados obtidos, tal como diâmetro de partícula de 5,94 micrômetros e potencial Zeta de $+7,03 \mathrm{mV}$, mostraram que a técnica utilizada para obtenção destas micropartículas foi satisfatória e possui potencial para aplicação na indústria de alimentos.
\end{abstract}

\section{INTRODUÇÃO}

Nas últimas décadas existe uma atenção direcionada para alimentos com propriedades funcionais importantes, o que tem impulsionado a indústria alimentícia a produzir alimentos com esse potencial. Entre os compostos com propriedades funcionais, encontram-se os antioxidantes, dentre eles as antocianinas (pigmentos naturais, parcialmente hidrossolúveis), as quais ajudam a proteger o organismo humano contra o estresse oxidativo que está associado a um aumento da incidência de câncer e outras doenças degenerativas (Scalbert \& Williamson, 2000 apud Moreira, 2007). As antocianinas estão presentes em vários produtos hortifrutigranjeiros. O jambo, objeto deste trabalho, é um deles. Apesar disso, seu uso como aditivo alimentício ainda está restrito em função de limitações como sazonalidade e a baixa estabilidade apresentada em algumas condições de processamento como temperatura, luz e oxigênio (Stringheta, 1991).

Os desafios estão, portanto, direcionados na busca de técnicas que façam com que este potencial antioxidante das antocianinas permaneça ativo e biodisponível não só após processamento e armazenamento, mas, também, que possa ser usado como veículo para a liberação de bioativos e micronutrientes em condições e níveis adequados. 


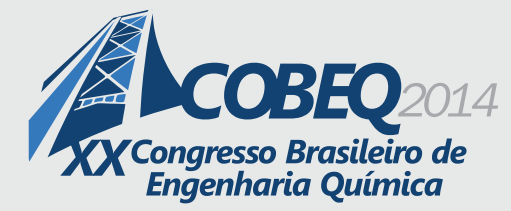

Dentro deste contexto, situam-se as técnicas de microencapsulação. $\mathrm{Na}$ indústria de alimentos, a microencapsulação é utilizada para fornecer ingredientes líquidos e sólidos estáveis, para preservar odores, fragrâncias, compostos bioativos, proteger os ingredientes encapsulados contra oxidação química ou de fatores do ambiente, dentre outros. Em algumas técnicas, a cápsula pode ser projetada para liberar lentamente o produto com o passar do tempo ou até que determinada condição físico-química seja alcançada (Thies, 1995). Por isso, as microcápsulas possuem um forte valor agregado com propriedades variadas e difíceis de controlar todas ao mesmo tempo.

A polimerização interfacial, técnica utilizada neste trabalho, é um método químico de microencapsulação utilizado para encapsular substâncias que têm em sua molécula uma parte hidrofílica e uma parte hidrofóbica, caso das antocianinas. Ela é formada por uma etapa de emulsificação seguida de uma etapa reativa. As reações ocorrem normalmente à temperatura ambiente.

Estudos anteriores mostraram que o extrato do corante do jambo (fruto de casca avermelhada e polpa branca abundante na região nordeste, contendo uma única grande semente de formato esférico em seu interior) possui uma atividade antioxidante superior a $85 \%$ e os resultados obtidos para a composição de fenólicos totais na casca indicam a possibilidade de sua utilização como fonte de antioxidantes naturais. (Azevedo, 2010).

Este trabalho teve como objetivo principal propor uma técnica de microencapsulação para preservar e armazenar por mais tempo as antocianinas presentes no extrato do corante do jambo vermelho.

\section{MATERIAIS E MÉTODOS}

\subsection{Materiais}

Quitosana micronizada de baixa massa molar (material de parede), - Sigma-Aldrich; Tween 80 - Oxiteno, Span 80 - Sigma-Aldrich, Isocianato (MDI) (agente reticulante) - SigmaAldrich; óleo de soja comercial, extrato do corante do jambo, antocianina Cyanidin-3-glucoside, Sigma- Aldrich, membranas de diálise, tampão fosfato padronizado - Sigma-Aldrich, ácido acético glacial, acetona, etanol, água destilada.

\subsection{Métodos}

2.2.1 Preparação das microcápsulas: A preparação das microcápsulas envolve duas etapas: a) Obtenção da microcápsula em "branco" ou "vazia"; b) Inserção do ativo dentro da microcápsula obtida. O método empregado para a obtenção das microcápsulas foi a polimerização interfacial precedida por uma etapa de emulsificação e evaporação do solvente. $O$ processo foi realizado em batelada sob agitação contínua e com controle da temperatura. Todos os experimentos foram feitos em triplicata. A polimerização interfacial utiliza uma solução aquosa e outra orgânica preparadas isoladamente e, em seguida, misturadas com agitação mecânica intensa para garantir a 


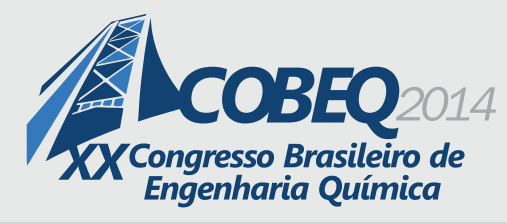

formação das microcápsulas por meio da existência de uma fase hidrofílica (solução aquosa) e uma hidrofóbica (solução orgânica). Após a obtenção desta microcápsula vazia, a fase seguinte do processo consiste em inserir o ativo no interior da mesma e caracterizá-la.

$\mathrm{O}$ planejamento experimental utilizado neste trabalho foi do tipo $2^{\mathrm{k}}$, onde $\mathrm{K}$ é o número de variáveis independentes: quantidade de quitosana, quantidade de óleo e quantidade de isocianato (MDI). O diâmetro da partícula foi escolhido como variável resposta por ser um dos fatores mais relevantes quando se quer delinear o uso em alimentos.

Com base no planejamento experimental proposto, foram realizados 8 ensaios com três repetições no ponto central (ensaios 9, 10 e 11). As variáveis independentes, em dois níveis, foram arranjadas segundo as 8 combinações previstas pelo planejamento experimental e no nível médio correspondente ao ponto central. A matriz experimental baseada no planejamento fatorial encontra-se ilustrada na Tabela 1.

Tabela 1. - Planejamento experimental $2^{3}$ - matriz experimental e variável resposta

\begin{tabular}{|c|c|c|c|c|}
\hline Ensaio & Quitosana $(\mathbf{g})$ & Óleo $(\mathbf{m L})$ & Isocianato $(\mathbf{g})$ & Diâmetro médio $(\boldsymbol{\mu m})$ \\
\hline 1 & -1 & -1 & -1 & 4,15 \\
\hline 2 & -1 & -1 & 1 & 52,06 \\
\hline 3 & -1 & 1 & -1 & 150,40 \\
\hline 4 & -1 & 1 & 1 & 19,66 \\
\hline 5 & 1 & -1 & -1 & 5,94 \\
\hline 6 & 1 & -1 & 1 & 22,63 \\
\hline 7 & 1 & 1 & -1 & 7,11 \\
\hline 8 & 1 & 1 & 1 & 16,19 \\
\hline 9 & 0 & 0 & 0 & 16,73 \\
\hline 10 & 0 & 0 & 0 & 23,36 \\
\hline 11 & 0 & 0 & 0 & 14,61 \\
\hline
\end{tabular}

Com base em experimentos preliminares, foram definidos os níveis das variáveis independentes, apresentados na Tabela 2.

Tabela 2 -. Níveis das variáveis independentes

\begin{tabular}{|c|c|c|c|}
\hline & $\mathbf{- 1}$ & $\mathbf{0}$ & $\mathbf{1}$ \\
\hline Quitosana (em gramas) & 0,5 & 0,75 & 1 \\
\hline Óleo de soja (em mililitros) & 1,5 & 2,5 & 3,5 \\
\hline Isocianato (MDI) (em gramas) & 0,1 & 0,25 & 0,4 \\
\hline
\end{tabular}

Estes ensaios foram realizados sem adição do material a ser encapsulado, pretendendo-se a partir da análise estatística dos resultados para a variável resposta, encontrar as condições que promovam a obtenção de partículas com menor diâmetro. 


\section{AcoseQ \\ Congresso Brasileiro de \\ Engenharia Química}

19 a 22 de outubro de 2014

Florianópolis/SC

Uma solução hidrofílica e outra hidrofóbica foram preparadas, mantendo-se para cada experimento as quantidades de quitosana, óleo de soja e isocianato definidas na matriz experimental. Para a solução aquosa, utilizaram-se os seguintes materiais: Quitosana, Tween 80, Água destilada, Ácido Acético. Para a solução oleosa, utilizou-se: Span 80, Isocianato, Óleo de soja, Acetona. Para cada ensaio realizado, as duas fases são misturadas no reator sob agitação e aquecimento controlados. A análise estatística foi realizada usando o software STATISTICA (Statsoftware, USA). Em seguida foram realizados ensaios em triplicata com estas condições adicionando-se como princípio ativo, o extrato de jambo ou o padrão de antocianina.

\subsubsection{Caracterização das micropartículas:}

Tamanho médio e distribuição de tamanho: A distribuição granulométrica das micropartículas obtidas foi determinada em aparelho Malvern Mastersizer/E (Malvern Instruments, Inglaterra). O tamanho médio das partículas foi expresso com o diâmetro médio de Sauter $\left(\mathrm{D}_{3,2}\right)$.

Morfologia: A análise morfológica das micropartículas foi realizada utilizando Microscopia Eletrônica de Varredura (MEV) e microscopia óptica.

Potencial Zeta: O Potencial Zeta é uma medida da estabilidade de uma dispersão. Quanto mais elevado seu valor numérico, mais estável é a dispersão. Neste trabalho as microcápsulas foram dispersas em água deionizada a $\mathrm{pH}$ 6,0 e a carga superficial foi medida por laser doppler anemômetro usando o Zetamaster (Malvern, UK).

\section{RESULTADOS E DISCUSSÕES}

\subsection{Ensaios Preliminares e Planejamento Experimental}

A partir da análise estatística dos resultados avaliou-se a influência das variáveis independentes na obtenção das micropartículas, bem como se propôs um modelo que representasse o diâmetro médio em função destas variáveis e das interações entre as mesmas. Os resultados do planejamento experimental se encontram na Tabela 1.

Estes dados foram submetidos a uma regressão linear utilizando o Statistica 7.0, onde foi possível calcular os efeitos de cada variável (Tabela 3).

Tabela 3 - Fatores e níveis para as variáveis codificadas definidas

\begin{tabular}{|c|c|c|c|}
\hline & $\mathbf{- 1}$ & $\mathbf{0}$ & $\mathbf{1}$ \\
\hline Quitosana $(\mathrm{g})$ & 0,5 & 0,75 & 1 \\
\hline Óleo de soja (mL) & 1,5 & 2,5 & 3,5 \\
\hline Isocianato (MDI) $(\mathrm{g})$ & 0,1 & 0,25 & 0,4 \\
\hline
\end{tabular}

A variável quantidade de quitosana foi a estatisticamente mais significativa para um nível de $95 \%$ de confiança. Isto pode ser constatado através do gráfico de Pareto (Figura 1). 


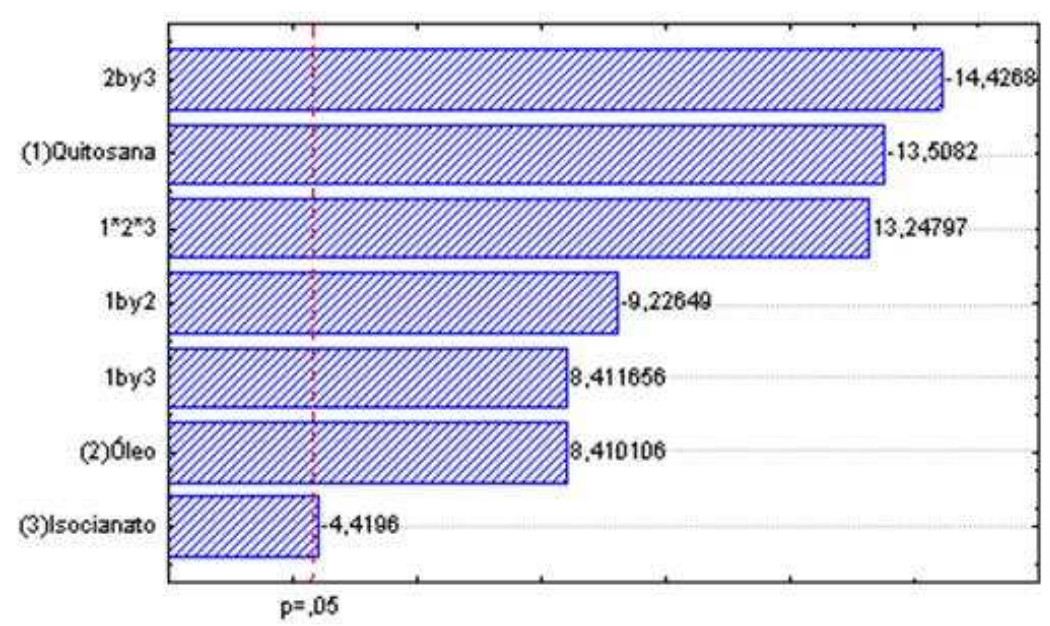

Figura: 1 - Gráfico de Pareto

Com a regressão dos dados, obteve-se um modelo matemático para o cálculo do diâmetro da partícula (Equação 1):

Diâmetro $=30,2582-21,8000 *$ quitosana $+13,5725 *$ oleo $-7,1325 *$ Isocianato $-14,8900 *$ quitosana $*$ oleo $+13,570 *$ quitosana $*$ isocianato $-23,2825 *$ oleo $*$ isocianato $+21,3800 *$ quitosana $*$ isocianato $*$ oleo

(1)

$\mathrm{O}$ coeficiente de correlação $\mathrm{R}^{2}$ ajustado para o modelo apresentado pela Equação 1 foi de 0,96366, o que demonstra uma regressão satisfatória.

\section{2-Tamanho médio e distribuição de tamanho}

Foram realizadas análises de tamanho médio e distribuição de tamanho para todos os ensaios de micropartículas de quitosana sem a incorporação do ativo. $\mathrm{O}$ ensaio 5 apresentou a melhor condição operacional e portanto, todos os resultados abaixo apresentados referem-se a este ensaio. A Figura 2 apresenta o resultado da distribuição de tamanho e índice de dispersão para estas condições.

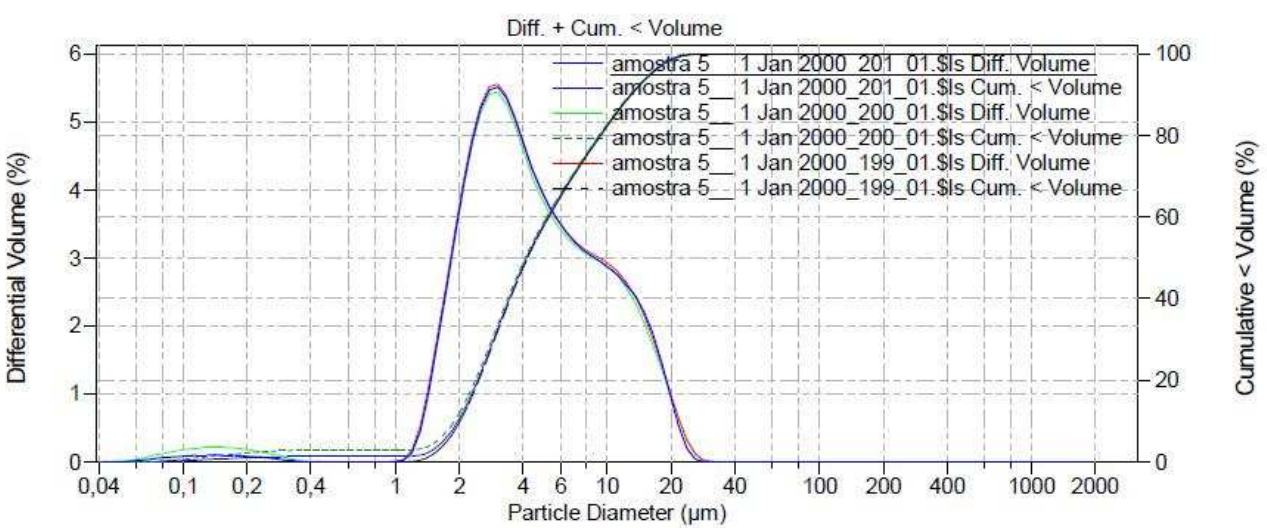

Figura 2 - Tamanho médio e distribuição de tamanho das micropartículas de quitosana/MDI (ensaio 5) 
Os valores encontrados $(5,920 \mu \mathrm{m}, 5,868 \mu \mathrm{m}$ e $6,019 \mu \mathrm{m})$ são satisfatórios para este trabalho, pois apresentam diâmetro compatível com o utilizado na indústria de alimentos e são superiores aos encontrados na literatura $(300 \mathrm{~nm}$ e $600 \mathrm{~nm})$, que também utilizaram quitosana como material de parede (Alvarez-Román et al., 2001; Alvarez-Román et al., 2004; Olvera-Martínez et al., 2005; Jiménez et al., 2004; Verna et al., 2003) .

\section{3-Morfologia}

A microscopia ótica foi utilizada para verificar a formação de micropartículas em suspensão durante os ensaios. As Figuras 3a e 3b mostram a dispersão das micropartículas em formação sem e com incidência direta de feixe de luz de fibra óptica respectivamente. Quando as partículas estão formadas não permitem a penetração da luz, apresentando-se sólidas.

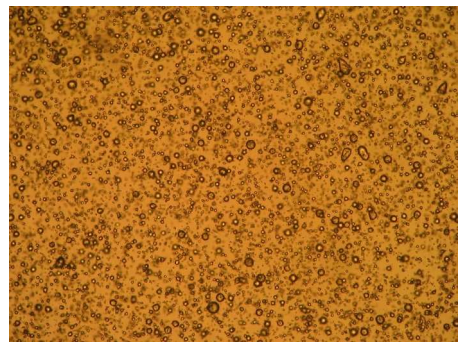

(a)Micropartículas sem luz polarizada

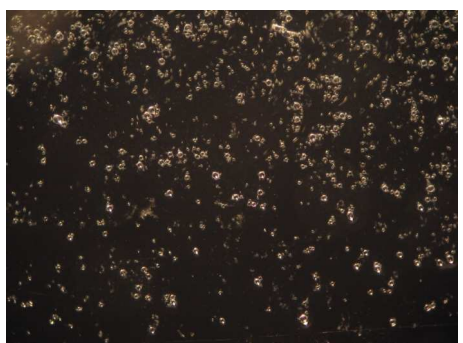

(b)Micropartículas sob luz polarizada

Figura 3 - Microscopia ótica das micropartículas de quitosana/MDI (100x)

A microscopia eletrônica de varredura mostra a morfologia das partículas vazias e das partículas contendo o extrato de jambo encapsulado (Figura 4).

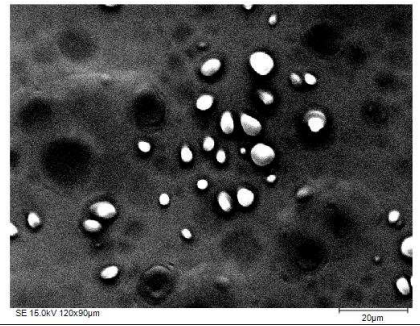

Micropartículas vazias (1000x)

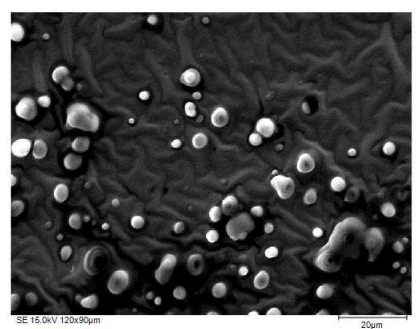

Micropartículas contendo extrato de jambo (1000x)

Figura 4 - Microscopia eletrônica de varredura das partículas com o sem extrato de jambo.

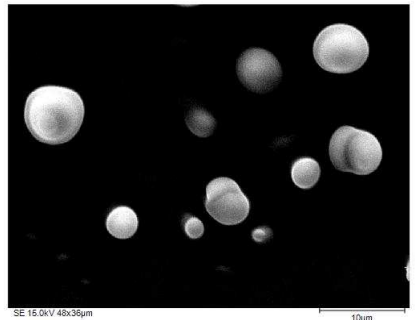

Micropartículas vazias (2500x)

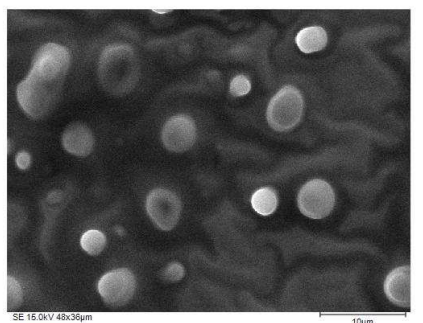

Micropartículas contendo extrato de jambo (2500x) 
Como observado nas fotos da Figura 4, as micropartículas tem formato predominantemente arredondado e pouco variável, com granulometria compatível com a que foi determinada por granulometria laser. Além disso, apresentam morfologia semelhante quando se comparam as partículas vazias com as que têm o extrato microencapsulado.

\section{4-Potencial Zeta}

O potencial Zeta das micropartículas vazias foi determinado através da média de quinze repetições sendo (Figura 5). Observa-se que as partículas têm carga superficial positiva, da ordem de $+7,03 \mathrm{mV}$ (amostra do ensaio 5). O potencial Zeta positivo encontrado na superfície das partículas é devido às características catiônicas das cadeias de quitosana em $\mathrm{pH}$ baixo. Com um aumento na quantidade de quitosana, há um excesso de íons $\mathrm{NH}_{3}{ }^{+}$, em relação aos grupamentos $\mathrm{COO}^{-}$o que resulta em micropartículas com valores maiores de potencial Zeta, tal qual estabeleceu Aouada, (2009). Conclui-se então que a emulsão formada neste trabalho é estável.

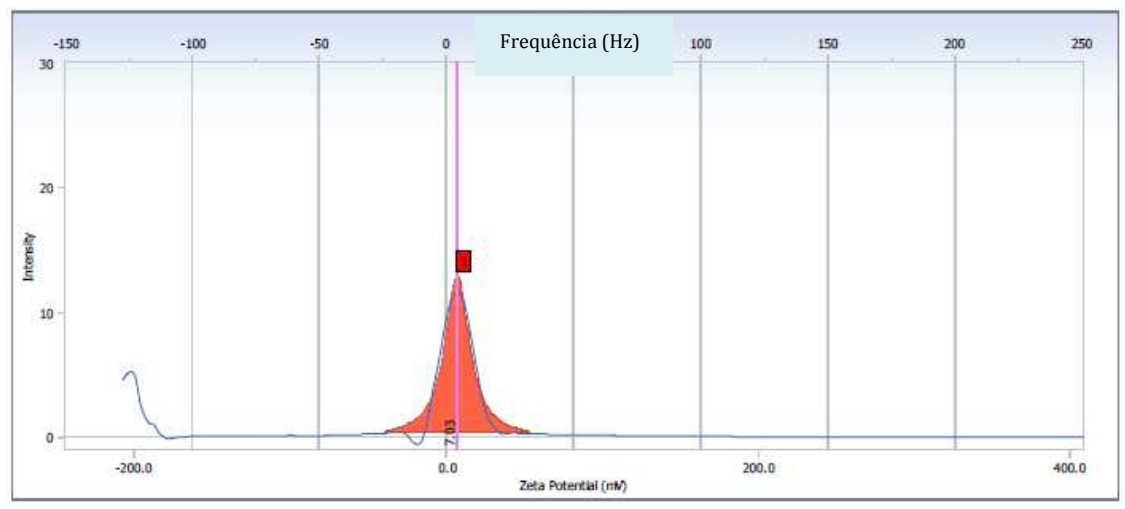

Figura 5 - Potencial Zeta das micropartículas vazias

\section{CONCLUSÕES}

Este trabalho teve como objetivo principal propor uma técnica de microencapsulação para preservar e armazenar por mais tempo as antocianinas presentes no extrato do jambo vermelho.

Foram obtidas microcápsulas vazias de quitosana e microcápsulas contendo o corante do extrato do jambo utilizando a técnica de polimerização interfacial.

A morfologia obtida indicou predominantemente característica esférica com pouca variação, o que indica boa reprodutibilidade do trabalho, aplicável em maior escala. A obtenção das microcápsulas com tamanhos diferentes foi satisfatória para posterior aplicação em diferentes áreas, principalmente na indústria de alimentos. 
O diâmetro obtido com as condições ideais de ensaio apresentou-se satisfatório para a indústria de alimentos, que em geral, utiliza partículas da ordem de 2 a $100 \mu \mathrm{m}$, dependendo da aplicação. O potencial Zeta apontou que as emulsões com e sem o extrato são estáveis, o que favorece boa vida de prateleira.

A microencapsulação das antocianinas do corante do extrato do jambo pode ser portanto, de grande utilidade para a indústria alimentícia por ser ainda pouco explorada.

\section{REFERENCIAS}

ALVAREZ-ROMÁN, R. et al., Biodegradable polymer nanocapsules containing a sunscreen agent: preparation and photoprotection. European Journal of Pharmaceutics and Biopharmaceutics, v. 52, p. 191-195, 2001.

ALVAREZ-ROMÁN, R. et al,. Skin penetration and distribution of polymeric nanoparticles. Journal of Controlled Release, v. 99, p. 53-62, 2004.

AZEVEDO, J. C. S. de. Estratégias de Obtenção do Corante do Jambo-Vermelho (Sysygium malaccense) e Avaliação de sua Funcionalidade. Dissertação (Mestrado). PPGEQ-UFRN. 2010.

AOUADA, M.R.M. Aplicação de nanopartículas em filmes utilizados em embalagens para alimentos Tese (Doutorado). São Carlos: UFSCAR, 2009.

COSTA NETO, B.P. Desenvolvimento de sistemas micro e nanoestruturados de quitosana/MDI para aplicações cosméticas. Dissertação (Mestrado). PPGEQ-UFRN. 2010.

JIMÉNEZ, M. M et al., M. C. Influence of encapsulation on the in vitro percutaneous absorption of octyl methoxycinnamate. International. Journal of Pharmaceutics, v. 272, p. 45-55, 2004.

MOREIRA, G. E. G. Obtenção e caracterização de extrato microencapsulado de resíduo agroindustrial de acerola. 72p. Dissertação(Mestrado), PPGEQ-UFRN. 2007.

MÜLLER-GOYMAN, C.C. Physicochemical characterization of colloidal drug delivery systems such as reverse micelles, vesicles, liquid crystals and a nanoparticles for topical administration. European Journal of Pharmaceuticals Biopharmaceuticals, v. 58, p. 343-346, 2004.

OLVERA-MATÍNEZ, B. et al., Preparation of polymeric nanocapsules containing octy methoxycinnamate by the emulsification-diffusion technique: penetration across the stratum corneum. Journal of Pharmaceutics Sciences, v. 94, n.7, p. 1552-1559, 2005.

SCALBERT, A.; WILLIAMSON, G. Dietary intake and bioavailability of polyphenols. Journal of Nutrition, v.130, n.8 (supl.), 2000.

STRINGHETA, P.C., Identificação da Estrutura e Estudos da Estabilidade das Antocianinas Extraídas da Inflorescência do Capim Gordura. Tese (Doutorado) em Ciência dos Alimentos. Unicamp. Brasil, 1991.

THIES, C. How to make microcapsules. St Louis, Missouri: Lecture and Laboratory, 1995

VERNA et al., Particle size of liposomes influnces dermal delivery of substances into skin. Internacional. Journal of Pharmaceutics, v. 258, p. 141-151, 2003. 